\title{
Low Frequency Raman Scattering from Acoustic Phonons Confined in ZnO Nanoparticles
}

\author{
Harish Kumar Yadav, ${ }^{1}$ Vinay Gupta, ${ }^{1}$ K. Sreenivas, ${ }^{1}$ S. P. Singh, ${ }^{2}$ B. Sundarakannan, ${ }^{3}$ and R. S. Katiyar ${ }^{3, *}$ \\ ${ }^{1}$ Department of Physics and Astrophysics, University of Delhi, Delhi-110007, India \\ ${ }^{2}$ National Physical Laboratory, Dr. K. S. Krishnan Marg, New Delhi-110012, India \\ ${ }^{3}$ Department of Physics, University of Puerto Rico, San Juan, Puerto Rico 00931-3343, USA
}

(Received 6 April 2006; published 25 August 2006)

\begin{abstract}
We report here the first observation of the low frequency Raman scattering from acoustic phonons in semiconducting zinc oxide $(\mathrm{ZnO})$ nanoparticles without embedding in any solid matrix. $\mathrm{ZnO}$ nanoparticles (size 5-10 nm) with nearly spherical shape have been synthesized using a chemical route. A shift in the phonon peaks toward higher frequencies along with broadening was observed with a decrease in particle size. The size dependence of the acoustic phonons in $\mathrm{ZnO}$ nanoparticles is explained using Lamb's theory that predicts the vibrational frequencies of a homogeneous elastic body of spherical shape. Our results show that the observed low frequency Raman scattering originates from the spherical $(l=0)$ and quadrupolar vibrations $(l=2)$ of the spheroidal mode due to the confinement of acoustic vibrations in $\mathrm{ZnO}$ nanoparticles.
\end{abstract}

DOI: 10.1103/PhysRevLett.97.085502

Introduction. - Semiconductor nanoparticles have been the subject of intense investigation because of their extraordinary electrical and optical properties at the nanoscale, which make them promising candidates for various applications [1-14]. The nanoparticles exhibit a characteristic change in electronic structure due to confinement of charge carriers and modify the vibrational phonon spectrum to size dependent discrete lines [2]. ZnO nanoparticles are among one of the compound semiconductors that show quantum confinement effect and possess extraordinary optical and electronic properties useful for application in electro-optic devices and UV lasers [3,4]. Photoluminescence properties $[4,5]$ and the confinement effect on excitons and optical phonons $[3,6]$ in $\mathrm{ZnO}$ nano particles have been investigated extensively. Raman spectroscopy is a powerful technique for investigating the vibrational phonon spectra of these systems. Resonant ultrasound spectroscopy is also useful for studying the mechanical resonant response of solids, but it is primarily used to determine their elastic moduli with high accuracy $[7,8]$. The size dependent Raman shift and the broadening of the optical phonon peaks in $\mathrm{ZnO}$ nanoparticles are routinely observed $[3,6]$.

Confined acoustic phonons have been observed in single element $\mathrm{Ag}, \mathrm{Au}$, and $\mathrm{Si}$ nanoparticles embedded in $\mathrm{SiO}_{2}$ thin film [9-11], and compound semiconductors $\mathrm{CdS}$, $\mathrm{CdSe}$, and $\mathrm{CdS}_{x} \mathrm{Se}_{1-x}$ nanoparticles present in a glass matrix [12-14]. A significant deviation in the low frequency mode was reported in comparison to the theoretically calculated value using Lamb's theory under stressfree boundary conditions $[15,16]$. Verma et al. modified Lamb's theory considering the influence of a solid matrix on the acoustic modes of embedded nanoparticles and showed good agreement between the observed modes and theory [14]. However, to the best of our knowledge, no result has been reported on the confinement of acoustic phonon in $\mathrm{ZnO}$ nanoparticles. In the present work, we
PACS numbers: 63.22.+m, 61.46.Df, 78.30.Fs, 81.07.Wx

report the first observation on confined acoustic phonons in $\mathrm{ZnO}$ nanoparticles using low frequency Raman spectroscopy. The influence of particle size on the acoustic mode has been studied using Lamb's theory under stressfree boundary conditions.

Experiment. - $\mathrm{ZnO}$ nanoparticles were synthesized using the dehydration property of alcohol at room temperature [17]. Zinc acetate dihydrate $(0.01 \mathrm{M})$ was dissolved in $75 \mathrm{ml}$ of ethanol at $75^{\circ} \mathrm{C}$ to obtain a clear solution. Lithium hydroxide monohydrate $(0.014 \mathrm{M})$ was dissolved in $50 \mathrm{ml}$ of ethanol using an ultrasonic bath. The hydroxide solution was added dropwise to the metal ion solution under vigorous stirring and was allowed to age for varying duration to obtain nanoparticles of different sizes. Nanoparticles were recovered by successive precipitation using $n$-heptane (ratio 1:3) and centrifugation. The samples prepared with aging times $0.25,1,2$, and $5 \mathrm{~h}$ are named as A, $\mathrm{B}, \mathrm{C}$, and $\mathrm{D}$, respectively.

$\mathrm{ZnO}$ nanoparticles were characterized by $\mathrm{x}$-ray diffraction (Siemens D5000) and a transmission electron microscope (Zeiss 922) for crystallographic orientation, shape, and size determination. The micro-Raman scattering measurements were performed in the backscattering geometry using a Jobin-Yvon T64000 triple monochromator equipped with a charge coupled device detector. The polarized light from a coherent INNOVA $99 \mathrm{Ar}^{+}$laser $(\lambda=$ $514.5 \mathrm{~nm}$ ) was focused to $2 \mu \mathrm{m}$ with $80 \times$ objective in an Olympus microscope.

Results. - The x-ray diffraction (XRD) peak profile of $\mathrm{ZnO}$ nanoparticles prepared with different aging time is shown in Fig. 1. The peak positions coincide with the Joint Committee on Powder Diffraction Standards data for the wurtzite $\mathrm{ZnO}$ structure, and the peak broadening suggests the formation of nanosize $\mathrm{ZnO}$ powder. The average size of the nanoparticles calculated using the Debye-Scherrer formula from (102) reflection for samples A, B, C, and D, respectively, was 5.7, 6.7, 7.1, and $10.1 \mathrm{~nm}$. Transmission 


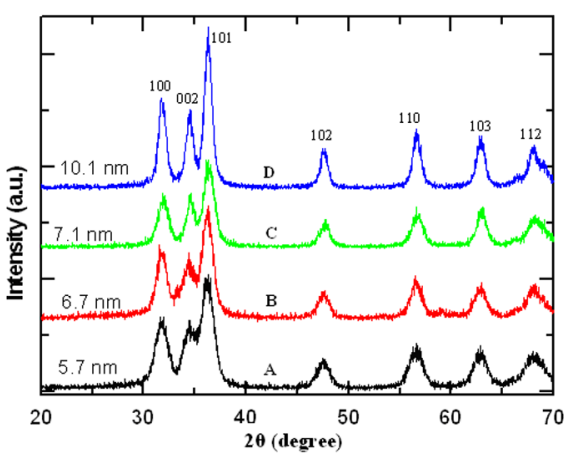

FIG. 1 (color online). X-ray diffraction pattern of the $\mathrm{ZnO}$ nanoparticles for $\mathrm{A}, \mathrm{B}, \mathrm{C}$, and $\mathrm{D}$.

electron microscope images [Figs. 2(a)-2(d)] indicate the formation of nearly spherical and monodispersed $\mathrm{ZnO}$ nanoparticles with sizes in the range 5-10 $\mathrm{nm}$ and are in agreement with the size obtained from the XRD data. Figure 3 shows the polarized low frequency Raman spectra of $\mathrm{ZnO}$ nanoparticles with varying size. All the samples exhibit one $E_{2}^{\text {low }}$ phonon peak at $\sim 100 \mathrm{~cm}^{-1}$ and two vibrational peaks (named peak 1 and peak 2, respectively) at around 10 and $22 \mathrm{~cm}^{-1}$. The intensity of both peaks 1 and 2 is found to be much higher in magnitude in comparison to that of the $E_{2}^{\text {low }}$ peak. As the size of the nanoparticles increased from sample A to sample D, peaks 1 and 2 shifted to lower frequency, along with a reduction in the peak widths and the separation between the two peaks (Fig. 3).

The low frequency Raman scattering in nanoparticles is considered to be due to an elastic vibration of the nanoparticle itself. A deconvolution was performed on all Raman spectra by assuming the phonons have Lorentzian shapes. It is important to point out that similar line shapes were observed in both parallel and cross polarization. The theoretical and experimental values of the position of

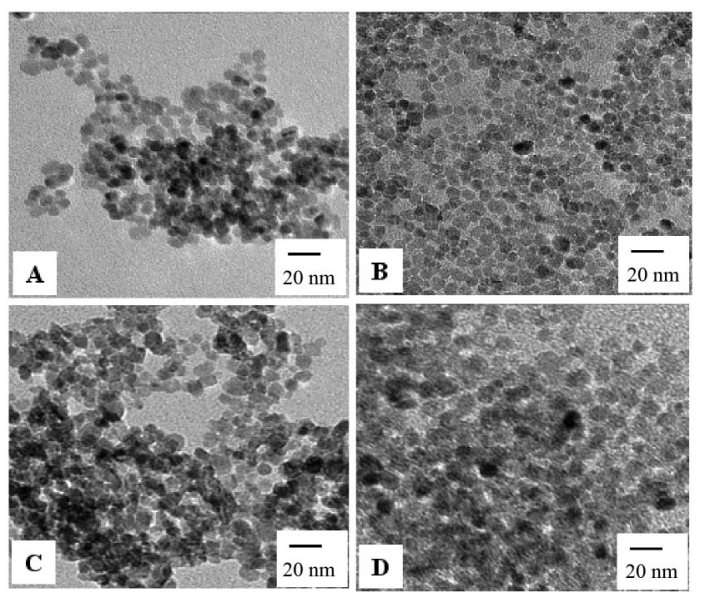

FIG. 2. Transmission electron microscope images of $\mathrm{ZnO}$ nanoparticles.

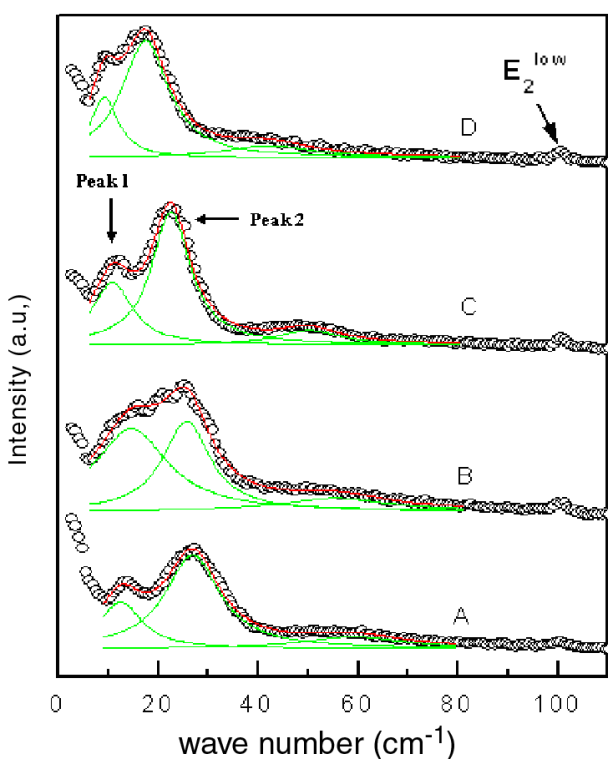

FIG. 3 (color online). Size dependent polarized low frequency Raman spectra of $\mathrm{ZnO}$ nanoparticles.

peaks 1 and 2 for $\mathrm{ZnO}$ nanoparticles of different sizes are given in Table I. In order to understand the influence of particle size, the observed phonon frequencies are plotted as a function of inverse particle diameter and are shown in Fig. 4 (square and circles). The position of the peaks 1 and 2 is found to be inversely proportional to the diameter $d$ of the nanoparticle and approaches zero as the size reaches the bulk value (Fig. 4). The observed low frequency modes are attributed to acoustic phonon confinement in the $\mathrm{ZnO}$ nanoparticles. Similar shifts in phonon modes with size have been reported for other nanoparticles embedded in a glass matrix [9-13]. The low frequency modes were fitted with the theoretically calculated eigenfrequencies of the confined acoustic modes using Lamb's theory under stressfree boundary condition because $\mathrm{ZnO}$ nanoparticles were synthesized without embedding in any solid matrix [16].

Lamb theoretically studied the vibrational spectra of a homogeneous elastic body [16]. The equation of motion for a spherical elastic body could be written as $[13,14]$

$$
\frac{\rho \partial^{2} \vec{D}}{\partial t^{2}}=(\lambda+\mu) \nabla(\nabla \cdot \vec{D})+\mu \nabla^{2} \vec{D}
$$

TABLE I. Experimental and theoretical values of the phonon modes for samples A-D.

\begin{tabular}{cccc}
\hline \hline Sample & $\begin{array}{c}\text { Diameter } \\
(\mathrm{nm})\end{array}$ & $\begin{array}{c}\omega_{01}\left(\mathrm{~cm}^{-1}\right) \\
(\text { peak 1) } \\
\text { expt/theor }\end{array}$ & $\begin{array}{c}\omega_{21}\left(\mathrm{~cm}^{-1}\right) \\
(\text { peak 2) } \\
\text { expt/theor }\end{array}$ \\
\hline A & 5.7 & $27.28 / 26.88$ & $13.22 / 12.46$ \\
B & 6.7 & $24.97 / 25.67$ & $14.33 / 14.60$ \\
C & 7.1 & $22.28 / 22.57$ & $11.58 / 10.83$ \\
D & 10.1 & $17.22 / 17.45$ & $9.77 / 9.25$ \\
\hline \hline
\end{tabular}


where $D$ is the displacement vector, $\lambda$ and $\mu$ are Lamb's constants, and $\rho$ is the mass density. Introducing proper scalar and vector potential, the solution of Eq. (1) gives two vibrational modes, namely, a spheroidal mode and a torsional mode. The eigenvalue equations for the spheroidal and torsional modes were derived as $[13,14]$

$$
\begin{gathered}
j_{l+1}(\eta)-\frac{l-1}{\eta} j_{1}(\eta)=0 \quad(l \geq 1) \quad \text { (torsional mode), } \\
\begin{aligned}
2\left[\eta^{2}+(l-1)(l+2)\left(\frac{\eta j_{1+1}(\eta)}{j_{1}(\eta)}-(l+1)\right)\right] & \frac{\xi j_{l+1}(\xi)}{j_{1}(\xi)}-\frac{\eta^{4}}{2}+(l-1)(2 l+1) \eta^{4} \\
& +\left[\eta^{2}-2 l(l-1)(l+2)\right] \frac{\eta j_{l+1}(\eta)}{j_{1}(\eta)}=0 \quad(l \geq 0) \quad \text { (spheroidal mode), }
\end{aligned}
\end{gathered}
$$

where $\eta$ and $\xi$ are dimensionless eigenvalues, and $j_{l}(\eta)$ is the spherical Bessel function of first kind. The frequencies of these modes were indexed with integers $n$ and $l$, known as the harmonic number and the angular momentum quantum number, respectively.

In order to analyze the results, selection rules and polarization properties of the low frequency acoustic modes were determined. The displacement vector $\mathbf{D}_{\mathrm{S}}$ and $\mathbf{D}_{\mathrm{T}}$ of the spheroidal and torsional vibrational modes, respectively, can be deduced from a scalar potential $\Phi$ and two vector potentials $\left(\mathbf{A}_{1}\right.$ and $\left.\mathbf{A}_{2}\right)$ as

$$
\begin{gathered}
\mathbf{D}_{\mathrm{S}}=\boldsymbol{\nabla \Phi}+\boldsymbol{\nabla} \times \boldsymbol{\nabla} \times \mathbf{A}_{1}, \\
\mathbf{D}_{\mathrm{T}}=\boldsymbol{\nabla} \times \mathbf{A}_{2} .
\end{gathered}
$$

From Eq. (4) it follows that $\mathbf{D}_{\mathrm{S}}$ has two independent directions, one along the radial unit vector and the other with a tangential component. However, torsional modes [Eq. (5)] have only an orthogonal direction and do not mix with the spheroidal mode. The spheroidal modes are characterized by $l \geq 0$, where $l=0$ is the spherical mode, $l=$ 1 is the dipolar mode, and $l=2$ is the quadrupolar mode. The torsional modes are characterized by $l \geq 1$ and has null displacement for $l=0$. The spheroidal modes for even $l$ (i.e., $l=0$ and 2 ) and the torsional modes for odd $l$ (i.e., $l=1$ and 3) are Raman active [12].

The Raman peak frequencies of the spheroidal mode can be expressed as

$$
\varpi_{l, n}^{\mathrm{sph}}=S_{l, n} \frac{\nu_{t}}{c d},
$$

where $S_{l, n}$ is the coefficient, $\nu_{t}$ is average transverse sound velocity of $\mathrm{ZnO}(\sim 2735 \mathrm{~m} / \mathrm{s}), d$ is diameter of the nanoparticle, and $c$ is velocity of light in vacuum. The coefficients $S_{l, n}$ depend strongly on the material through the ratio of the transverse to the longitudinal sound velocity $\left(\nu_{t} / \nu_{l}\right)$, and therefore the Raman peak frequency of the spherical and quadrupolar mode of spheroidal motion for $n=1$ is obtained from Eq. (4) as

$$
\begin{gathered}
\varpi_{l, n}^{\mathrm{sph}}=2.07 \frac{\nu_{t}}{c d} \quad \text { (spherical), } \\
\varpi_{l, n}^{\mathrm{sph}}=0.84 \frac{\nu_{t}}{c d} \quad \text { (quadrupolar). }
\end{gathered}
$$

The Raman shifts of both modes are proportional to the sound velocity and inversely proportional to the diameter (d) of nanoparticle. The theoretical values of frequencies for spheroidal modes for $l=0$ have been calculated at different values of " $d$ " both under the free and the fixed boundary condition, whereas that of the quadrupolar vibrations $(l=2)$ are calculated only under the stress-free boundary condition. Good agreement between the experimental data of low frequency acoustic phonon with theoretical calculations for spheroidal modes with $l=0$ and 2 as a function of diameter of $\mathrm{ZnO}$ nanoparticle was observed (Fig. 4). The theoretical values of frequencies for

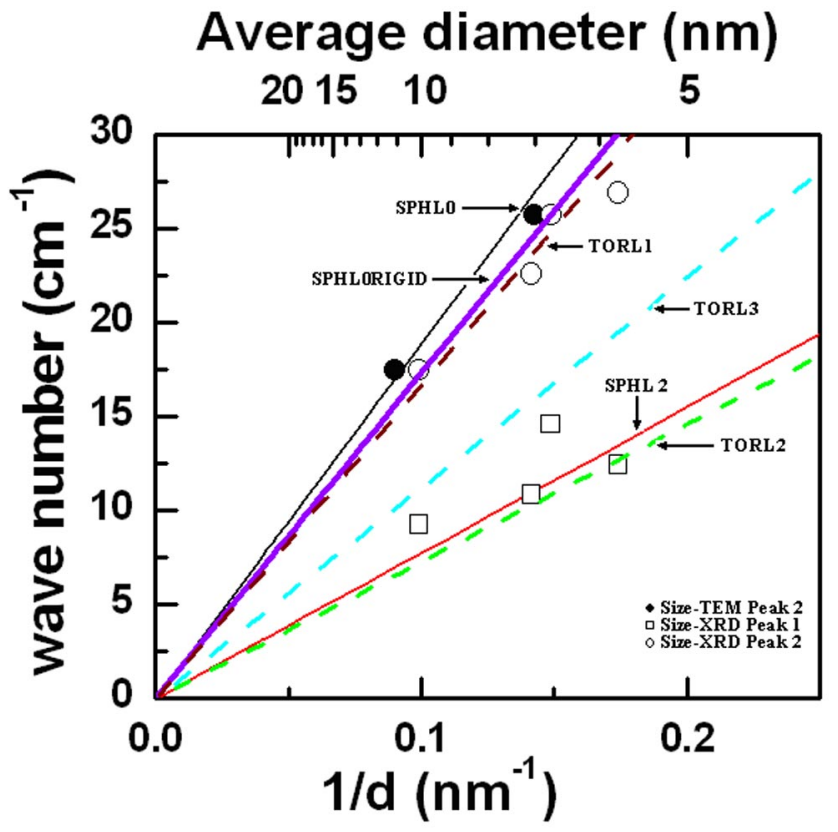

FIG. 4 (color online). Peak frequencies of polarized Raman spectra as a function of inverse particle diameter. The solid lines are calculated for spheroidal modes with $l=0$ (SPHL0) and $l=$ 2 (SPHL2) with free boundary conditions while $l=0$ (SPHLORIGID) with fixed boundary conditions. The dashed lines are calculated for torsional modes for $l=1$ (TORL1), $l=$ 2 (TORL2), and $l=3$ (TORL3). Squares and circles are the experimentally measured frequency with the sizes of nanoparticles estimated from XRD (open square and circle) and TEM (solid circles). 
torsional modes with $l=1,2$, and 3 were also calculated under the stress-free boundary condition and are included in Fig. 4 (dashed lines).

The spheroidal mode with $l=0$ is purely radial and appears only in the parallel polarization, while the $l=2$ mode appears in both parallel and crossed polarization. The low frequency modes in the nanoparticles were observed in both parallel and cross polarizations, and are primarily assigned to the spherical and the quadrupolar vibrations, $l=0$ and $l=2$ for $n=1$. It may be noted from Fig. 4 that the calculated Raman-shift of the torsional mode with $l=$ 1 (TORL1) is close to peak 2 (open circles); however, this mode is partially depolarized. Furthermore, peak 1 is found close to the calculated values for the torsional mode with $l=2$ (TORL2), but this mode is not Raman active. Therefore, it is concluded that the observed peaks in the low frequency Raman spectra originate from the spheroidal modes with $l=0$ and $l=2$ and are attributed to the confinement of acoustic phonons. The appearance of the $l=0$ mode in cross polarization may be due to a slight deviation in the assumption of the spherical shape for the synthesized nanoparticles or due to anisotropy in the velocity of sound along different crystallographic directions.

Saviot and Murray reported that the spheroidal modes can be classified into longitudinal (LA) and transverse (TA) modes [18]. The distinction between these modes is shown to be best when the ratio $v_{t} / v_{l}$ is small. The ratio $v_{t} / v_{l}$ is considerably high for $\mathrm{ZnO}$ nanoparticles $(\sim 0.8-2.04)$; therefore the assignment of the low frequency peaks to LA and TA is difficult. In a recent report, Kanehisa modified the selection rules, according to which only one peak corresponding to torsional mode with $l=2$ should appear under both parallel and cross polarization [19]. It is important to note that two peaks (1 and 2) were observed in the present study under both polarizations. A similar observation on the appearance of two peaks has been reported for embedded $\mathrm{CdS}_{x} \mathrm{Se}_{1-x}$ nanoparticles under parallel polarization [14] and the observed modes follow the selection rules reported by Duval [15]. However, further studies on nanoparticles of different materials without embedding in any solid matrix may resolve the controversy.

The theoretical values of eigenfrequencies were generally reported to be much higher than those observed experimentally in the nanoparticle embedded in glass matrix [11], and the discrepancy is attributed to the assumption of a stress-free boundary condition for the composite system. However, Verma et al. showed good agreement between the theoretical and the experimental values for $\mathrm{CdS}_{x} \mathrm{Se}_{1-x}$ nanoparticles embedded in silicate matrix after modifying Lamb's theory [14]. In the present study good agreement between the observed low frequency modes with the calculated value for spheroidal modes $(l=0$ and $l=2)$ indicates that the assumption of $\mathrm{ZnO}$ nanoparticles as an elastic sphere with a stress-free surface boundary condition is justified.
Conclusion.-We have observed for the first time Raman scattering from confined acoustic phonons in $\mathrm{ZnO}$ nanoparticles (size 5-10 $\mathrm{nm}$ ) without embedding in any solid matrix. The spherical form of $\mathrm{ZnO}$ nanoparticles allow us to consider them as isolated homogeneous elastic spheres and the observed size dependence of acoustic modes were explained in the light of Lamb's theory under the stress-free boundary condition. The observed low frequency peaks were assigned to the spheroidal modes $l=0$ and $l=2$, which shifted towards lower frequency with an increase in the diameter of the $\mathrm{ZnO}$ nanoparticles. Although Lamb's theory could well explain the observed size dependence of low frequency mode, further studies are required to understand the confined acoustic phonons in free nanoparticles.

The work is partially supported by the DST, CSIR, India and the DOD, USA. H. K.Y. is also thankful to UGC, India. The authors acknowledge Professor Vikram Kumar and Professor K. P. Jain for fruitful discussion and Ms. Jaya Chaudhary for helping in the present work.

*Corresponding author. Electronic address: rkatiyar@uprrp.edu

[1] A. P. Alivisatos, J. Phys. Chem. 100, 13226 (1996).

[2] F. W. Wise, Acc. Chem. Res. 33, 773 (2000).

[3] M. Rajalakshmi, A. K. Arora, B. S. Bendre, and S. Mahamuni, J. Appl. Phys. 87, 2445 (2000).

[4] C. L. Yang, J. N. Wang, W. K. Ge, L. Guo, S. H. Yang, and D. Z. Shen, J. Appl. Phys. 90, 4489 (2001).

[5] L. Guo, S. Yang, C. Yang, P. Yu, J. Wang, W. Ge, and G. K. L. Wong, Appl. Phys. Lett. 76, 2901 (2000).

[6] K. A. Alim, V. A. Fonoberov, and A. A. Balandin, Appl. Phys. Lett. 86, 053103 (2005).

[7] A. Migliori, J. L. Sarrao, W. M. Visscher, T. M. Bell, M. Lei, Z. Fisk, and R. G. Leisure, Physica (Amsterdam) 183B, 1 (1993).

[8] D. G. Isaak, J. D. Carnes, and O. L. Anderson, J. Acoust. Soc. Am. 104, 2200 (1998).

[9] M. Fujii, T. Nagareda, S. Hayashi, and K. Yamamoto, Phys. Rev. B 44, 6243 (1991).

[10] M. Fujii, T. Nagareda, S. Hayashi, and K. Yamamoto, J. Phys. Soc. Jpn. 61, 754 (1992).

[11] M. Fujii, Y. Kanzawa, S. Hayashi, and K. Yamamoto, Phys. Rev. B 54, R8373 (1996).

[12] A. Tanaka, S. Onari, and T. Arai, Phys. Rev. B 47, 1237 (1993).

[13] A. Tanaka, S. Onari, and T. Arai, Phys. Rev. B 45, 6587 (1992).

[14] P. Verma, W. Cordts, G. Irmer, and J. Monecke, Phys. Rev. B 60, 5778 (1999).

[15] E. Duval, Phys. Rev. B 46, 5795 (1992).

[16] H. Lamb, Proc. London Math. Soc. 13, 187 (1882).

[17] L. Spanhel and M. A. Anderson, J. Am. Chem. Soc. 113, 2826 (1991).

[18] L. Saviot and D. B. Murray, Phys. Rev. B 72, 205433 (2005).

[19] M. Kanehisa, Phys. Rev. B 72, 241405(R) (2005). 